\title{
Spin dynamics of the quantum $X Y$ chain and ladder in a random field
}

\author{
M.E.S. Nunes ${ }^{\mathrm{a}}$, J.A. Plascak ${ }^{\mathrm{a}}$, J. Florencio ${ }^{\mathrm{b}, *}$ \\ ${ }^{a}$ Departamento de Física, ICEX, CP 702, Universidade Federal de Minas Gerais, \\ 30123-970 Belo Horizonte, Minas Gerais, Brazil \\ ${ }^{\mathrm{b}}$ Instituto de Física, Universidade Federal Fluminense, 24210-340 Niterói, Rio de Janeiro, Brazil
}

Received 25 March 2003; received in revised form 27 August 2003

\begin{abstract}
We investigate the Hamiltonian dynamics of two low-dimensional quantum spin systems in a random field, at the infinite-temperature limit: the $X Y$ chain and the two-leg $X Y$ ladder with interchain Ising interactions. We determine the longitudinal spin autocorrelation functions of the spin- $\frac{1}{2} X Y$ chain and ladder in the presence of disordered fields by using the method of recurrence relations. The first six basis vectors for the chain and the first four basis vectors for the ladder of the dynamic Hilbert spaces of $\sigma_{j}^{z}(t)$, as well as the corresponding recurrents and moments of the time-dependent autocorrelation function, are analytically computed for bimodal distributions of the fields. We did find a remarkable result in the disordered models. Cases with a fraction of $p$ sites under field $B_{B}$ and a fraction of $1-p$ sites under the field $B_{A}$ have the same longitudinal dynamics as those with $p$ sites under field $B_{A}$ and $1-p$ sites under the field $B_{B}$. We also find that both the $X Y$ chain and the two-leg $X Y$ ladder with Ising interchain coupling in the presence of random fields are sensitive to the percentage of disorder but not to the intensity of the fields. (C) 2003 Elsevier B.V. All rights reserved.
\end{abstract}

PACS: 75.40.Gb; 75.50.Lk; 75.10.Jm; 05.05.+q

Keywords: $X Y$ model; Spin dynamics; Method of recurrence relations; Continued fractions

\section{Introduction}

The quantum $X Y$ chain has attracted considerable attention during the last four decades not only as an interesting many-body theoretical problem but also due to

\footnotetext{
${ }^{*}$ Corresponding author.

E-mail addresses: eugenia@fisica.ufmg.br (M.E.S. Nunes), pla@fisica.ufmg.br (J.A. Plascak), jfj@iffuff.br (J. Florencio).
} 
its applicability to real quasi-one-dimensional compounds. The Hamiltonian of the one-dimensional spin- $\frac{1}{2} X Y$ model can be defined by

$$
H=\frac{1}{2} \sum_{i=1}^{N}\left(J_{x} \sigma_{i}^{x} \sigma_{i+1}^{x}+J_{y} \sigma_{i}^{y} \sigma_{i+1}^{y}\right)-\frac{1}{2} \sum_{i=1}^{N} B_{i} \sigma_{i}^{z},
$$

where $\sigma_{i}^{\alpha}(\alpha=x, y, z)$ are Pauli matrices on site $i, J_{x}$ and $J_{y}$ are nearest-neighbor coupling constants, $N$ is the number of sites, and $B_{i}$ is the field at site $i$. This model, with $B_{i}=0$, was introduced in 1961 and many of its properties have been determined exactly [1-4]. It has been successfully used to describe the dynamic behavior of quasi-one-dimensional compounds, such as $\mathrm{PrCl}_{3}$ [5] and $\mathrm{CsCoCl}_{3}$ [6]. Lieb et al. [1], who introduced the model, used the Jordan-Wigner transformation [7] to map the problem onto that of a system of non-interacting spinless fermions to find the exact energies of the model. Ever since, a good deal of work has appeared in the literature concerning mainly its thermodynamic properties. Katsura [2] solved the anisotropic $X Y$ model in the presence of a uniform magnetic field in the $z$ direction (i.e., $J_{x} \neq J_{y}$ and $B_{i}=B$ ) with the help of Jordan-Wigner transformation. He obtained exact results for the temperature and magnetic field dependence of various thermodynamics properties such as magnetization, susceptibility, and specific heat, among others. Suzuki [8] studied the effect of a staggered magnetic field $B_{i}$ by using a theorem to calculate the partition function exactly. A further extension on this model was done by Derzhko and Richter [9] who calculated some thermodynamic quantities for the quantum $X Y$ chain with random Lorentzian intersite interaction and transverse field, using the Jordan-Wigner transformation.

Regarding the dynamics of this model, Niemeijer [10] obtained the exact timedependent correlation function $C(t)=\left\langle\sigma_{j}^{z}(0) \sigma_{j}^{z}(t)\right\rangle$ at $T \rightarrow \infty$ and $B_{i}=0$ for the isotropic model $\left(J_{x}=J_{y}\right)$. The result is simply $C(t)=\left[J_{0}(2 t)\right]^{2}$, where $J_{0}$ is the zeroth order Bessel function. McCoy [11] studied the spatial spin pair correlation function along the $x, y$, and $z$ directions for different anisotropies in the limit of large number of sites. At $T \neq 0$, those correlation functions decay to zero exponentially as the number of spins $N \rightarrow \infty$. On the other hand, at $T=0$ the transverse correlation function decays exponentially to a non-zero value if its direction is along that of the stronger coupling, while the other transverse correlation function approaches zero exponentially as $N \rightarrow \infty$. In the isotropic case, all three correlation functions approach zero following power laws, as $N \rightarrow \infty$.

Barouch et al. [12-14] studied the time-dependent properties of the $z$-direction magnetization. They considered the $X Y$ model in thermal equilibrium at temperature $T$ in the presence of a uniform external magnetic field $B_{1}$. At $t=0$, the field is changed to some other value $B_{2}$, and $M_{z}(t)$ is then determined. The most interesting aspect is that if $B_{2}=0$, then $M_{z}(t) \neq 0$ as $t \rightarrow \infty$. They also examined the asymptotic behavior of the space-time dependent spin correlation function at $T=0$. Sur et al. [15] calculated the exact transverse autocorrelation function for finite chains containing 5,7, and 9 spins. Their analysis suggested that such autocorrelation function for the infinite chain is given by $\exp \left(-J^{2} t^{2}\right) / 4$. In fact, later on, Brandt and Jacoby [16] and also Capel and Perk [17] obtained independently the same Gaussian behavior. Florencio and 
Lee [18] investigated the dynamics of the one-dimensional $s=\frac{1}{2}$ isotropic $X Y$ model and the transverse Ising model in the high-temperature limit by using the method of recurrence relations. They found that the dynamic Hilbert spaces of the two models have the same structure, which leads to a similar dynamic behavior apart from a time scale.

Quantum models defined on a ladder structure have also been of considerable interest since they can be viewed as an interpolation between one- and two-dimensional systems. Physical realizations such as $(\mathrm{VO})_{2} \mathrm{P}_{2} \mathrm{O}_{7}$ [19] and $\mathrm{Cu}_{2}\left(\mathrm{C}_{5} \mathrm{H}_{12} \mathrm{~N}_{2}\right)_{2} \mathrm{Cl}_{4}$ [20] correspond in fact to quantum Heisenberg ladders. The full two-leg ladder HeisenbergIsing Hamiltonian can be written as

$$
\begin{aligned}
H= & \frac{1}{2} \sum_{i=1}^{N}\left(J_{x} \sigma_{i, 1}^{x} \sigma_{i+1,1}^{x}+J_{y} \sigma_{i, 1}^{y} \sigma_{i+1,1}^{y}+J_{z} \sigma_{i, 1}^{z} \sigma_{i+1,1}^{z}\right) \\
& +\frac{1}{2} \sum_{i=1}^{N}\left(J_{x} \sigma_{i, 2}^{x} \sigma_{i+1,2}^{x}+J_{y} \sigma_{i, 2}^{y} \sigma_{i+1,2}^{y}+J_{z} \sigma_{i, 2}^{z} \sigma_{i+1,2}^{z}\right) \\
& +\frac{1}{2} \sum_{i=1}^{N} I_{i} \sigma_{i, 1}^{z} \sigma_{i, 2}^{z}-\frac{1}{2} \sum_{i=1}^{N} B_{i, 1} \sigma_{i, 1}^{z}-\frac{1}{2} \sum_{i=1}^{N} B_{i, 2} \sigma_{i, 2}^{z},
\end{aligned}
$$

where subscripts 1 and 2 refer to chains 1 and $2, J_{\alpha}(\alpha=x, y, z)$ are exchange couplings, $I_{i}$ is the interchain Ising coupling, and $B_{i, 1}$ and $B_{i, 2}$ are external fields. Most of the theoretical work with spin ladders deals with ladders with Heisenberg or $X X Z$ interactions along the legs. Only a few of them concern the case where $J_{z}=0$, that is, the $X Y$-Ising ladder. Huber and Caille [21] studied the $X Y$-Ising ladder consisting of two chains, with $J_{x}=J_{y}$, connected by a uniform Ising interaction $I$. They used the Jordan-Wigner transformation to obtain the specific heat. Hikihara and Furusaki [22] numerically computed the spatial correlation functions along the $x$ and $z$ directions by using the density matrix and renormalization group approaches. Orignac and Giamarchi [23] treated anisotropic spin- $\frac{1}{2} X Y$ ladders in the presence of several types of weak random perturbations. They found that the effect of the disorder depends on whether or not the $X Y$ symmetry is preserved. More recently, Mélin et al. [24] considered ladders with a strong disorder in the antiferromagnetic interactions.

In this paper, we study the effects of an applied random field on the dynamic behavior at the infinite-temperature limit of the longitudinal autocorrelation function of the following spin- $\frac{1}{2}$ systems: (i) the $X Y$ model in one dimension, and (ii) the two-leg $X Y$-Ising ladder. In both cases, the fields $B_{i}$ are drawn independently according to bimodal distributions. The main theoretical tool we use is the method of recurrence relations [25-29], which has been successfully used to treat a variety of dynamic problems such as, the velocity autocorrelation function [30], interacting electron gas [31,32], quantum spin systems [18,33-35], classical diffusion [36-38], simple fluids [39], and ergodic theory [40], among others. We should stress that in the present work, we shall use an extension of the method of recurrence relations in order to properly account for the random fields. 
This paper is arranged as follows. In Section 2 we review the method of recurrence relations. In Section 3 that method is applied to study the dynamical behavior of the $X Y$ chain in a disordered field. Our study of the dynamics of the $X Y$-Ising ladder is presented in Section 4. Finally, in Section 5 we summarize our results.

\section{Method of recurrence relations}

Consider a system described by a Hamiltonian $H$. The time evolution of an operator $A$ is governed by

$$
\frac{\mathrm{d} A(t)}{\mathrm{d} t}=\mathrm{i} L A(t)
$$

where $L$ is the Liouville operator, defined by $L A=[H, A] \equiv H A-A H$. The solution to Eq. (3) can be cast as the following orthogonal expansion

$$
A(t)=\sum_{n=0}^{d-1} a_{n}(t) f_{n},
$$

where the $f_{n}$ 's are basis vectors spanning a $d$-dimensional Hilbert space $S$. In order to include an average over disorder, we now modify the original definition of the scalar product, that is, we define the scalar product in $S$ as the Kubo product [41] averaged over the random variables

$$
(X, Y)=\frac{1}{\beta} \int_{0}^{\beta} \mathrm{d} \lambda \overline{\left\langle X(\lambda) Y^{\dagger}\right\rangle}-\overline{\langle X\rangle\left\langle Y^{\dagger}\right\rangle},
$$

where $\overline{\langle\cdots\rangle}$ denotes an ensemble average and an average over all realizations of disorder. Here, $X$ and $Y$ are vectors defined in $S, \beta=1 / k_{B} T$ is the inverse temperature, and $X(\lambda)=\exp (\lambda H) X \exp (-\lambda H)$. In the high-temperature limit, $T \rightarrow \infty$, the scalar product reduces to

$$
(X, Y)=\frac{\overline{\operatorname{Tr} X Y^{\dagger}}}{\operatorname{Tr} 1},
$$

where $\operatorname{Tr} 1$ gives the number of states of the system.

By choosing $f_{0}=A(0)$, it follows that the remaining basis vectors can be generated by using the following recurrence relation:

$$
f_{n+1}=\mathrm{i} L f_{n}+\Delta_{n} f_{n-1},
$$

where $0 \leqslant n \leqslant d-1$. The quantity

$$
\Delta_{n}=\frac{\left(f_{n}, f_{n}\right)}{\left(f_{n-1}, f_{n-1}\right)}, \quad n \geqslant 1
$$

gives the relative norms of consecutive basis vectors, and is usually referred to as recurrent. By definition, $f_{-1} \equiv 0$ and $\Delta_{0} \equiv 1$.

The coefficients $a_{n}(t)$ s satisfy a second recurrence relation

$$
\Delta_{n+1} a_{n+1}(t)=-\frac{\mathrm{d} a_{n}(t)}{\mathrm{d} t}+a_{n-1}(t),
$$


where $0 \leqslant n \leqslant d-1$, and $a_{-1}(t) \equiv 0$. Notice that with the initial choice $f_{0}=A(0)$, it follows from Eq. (4) that $a_{0}(0)=1$, and $a_{n}(0)=0$ for $n \geqslant 1$. Hence, $a_{0}(t)$ represents the relaxation function of linear response theory. In the limit $T \rightarrow \infty, a_{0}(t)$ is simply the time-dependent autocorrelation function $C(t)$. The complete time evolution of $A(t)$ can thus be determined by using (RRI) and (RRII).

By applying the Laplace transform to (RRII), one obtains

$$
\begin{aligned}
& \Delta_{1} \alpha_{1}(z)=1-z \alpha_{0}(z), \quad n=0, \\
& \Delta_{n+1} \alpha_{n+1}(z)=-z \alpha_{n}(z)+\alpha_{n-1}(z), \quad n \geqslant 1,
\end{aligned}
$$

where

$$
\alpha_{n}(z)=\int_{0}^{\infty} \mathrm{e}^{-z t} a_{n}(t) \mathrm{d} t, \quad \operatorname{Re} z>0 .
$$

By using Eqs. (8) and (9), one obtains the continued fraction for $\alpha_{0}(z)$ :

$$
\alpha_{0}(z)=\frac{1}{z+\left(\Delta_{1} / z+\left(\Delta_{2} / z+\cdots\right)\right)} .
$$

Note that the $\Delta_{n}$ are the sole ingredients that enter the determination of the dynamic correlation functions. In addition, the knowledge of the $\Delta_{n}$ enables one to obtain the moments of the correlation function. In practice, only a few $\Delta_{n}$ can be determined analytically.

By working on the high-temperature limit, $T=\infty$, we managed to obtain the first six $\Delta_{n}$ pertaining to $\sigma_{j}^{z}(t)$ and Hamiltonian (1), and the first four $\Delta_{n}$ for Hamiltonian (2). From the $\Delta_{n}$, we determine the corresponding moments $\mu_{2 n}$ of the average time-dependent correlation function

$$
C(t)=\overline{\left\langle\sigma_{j}^{z}(0) \sigma_{j}^{z}(t)\right\rangle}=\sum_{k=0}^{\infty} \mu_{2 k} t^{2 k},
$$

where $\mu_{2 k}$ are defined by the following formula involving $2 k$ nested commutators,

$$
\mu_{2 k}=\frac{1}{2 k !}(-1)^{k} \overline{\left\langle\left[H,\left[H, \ldots\left[H, \sigma_{j}^{z}\right] \cdots\right]\right] \sigma_{j}^{z}\right\rangle} .
$$

It is also possible to compute the spectral function $\Phi(\omega)$ which is defined as the Fourier transform of the time-dependent autocorrelation function

$$
\Phi(\omega)=\int_{-\infty}^{\infty} \mathrm{e}^{\mathrm{i} \omega t} C_{0}(t) \mathrm{d} t
$$

This spectral function has the advantage of being accessible in experiments.

\section{Dynamics of the $X Y$ chain in a random field}

In this section, we focus on the spin autocorrelation function of the $X Y$ model in one dimension in the infinite-temperature limit. Hamiltonian (1) is given by the following, in the isotropic case $\left(J_{x}=J_{y}=J\right)$,

$$
H=\frac{J}{2} \sum_{i=1}^{N}\left(\sigma_{i}^{x} \sigma_{i+1}^{x}+\sigma_{i}^{y} \sigma_{i+1}^{y}\right)-\frac{1}{2} \sum_{i=1}^{N} B_{i} \sigma_{i}^{z},
$$


where the fields $B_{i}$ are randomly drawn according to the bimodal distribution

$$
\rho\left(\left\{B_{i}\right\}\right)=\prod_{i}^{N}\left[p \delta\left(B_{i}-B_{A}\right)+(1-p) \delta\left(B_{i}-B_{B}\right)\right]
$$

with $0 \leqslant p \leqslant 1$, where $p$ represents the probability of drawing $B_{A}$. Thus $p=0$ and $p=1$ correspond to the cases $B_{i}=B_{B}$ and $B_{i}=B_{A}$, respectively. We shall be interested in the cases where $B_{A}=0$ and $B_{B}=1.5 \mathrm{~J}$. The distribution $\rho$ is normalized to unity and the average of a given function $f\left(\left\{B_{i}\right\}\right)$ is obtained simply by using the integral

$$
\overline{f\left(\left\{B_{i}\right\}\right)}=\int_{-\infty}^{\infty} \rho\left(\left\{B_{i}\right\}\right) f\left(\left\{B_{i}\right\}\right) \prod_{i}^{N} \mathrm{~d} B_{i} .
$$

Since we are interested in the longitudinal correlation function in the limit of infinite temperature, Eq. (12), we express the time evolution of the tagged spin $\sigma_{j}^{z}$ as follows:

$$
\sigma_{j}^{z}(t)=\sum_{n=0}^{d-1} a_{n}(t) f_{n},
$$

where $f_{0}=\sigma_{j}^{z}(0)=\sigma_{j}^{z}$. Clearly, $\left(f_{0}, f_{0}\right)=\left(\sigma_{j}^{z}, \sigma_{j}^{z}\right)=1$. The other basis vectors are determined using the recurrence relation (RRI). Thus for $f_{1}$, we obtain

$$
f_{1}=J \sigma_{j}^{y} \sigma_{j+1}^{x}+J \sigma_{j-1}^{x} \sigma_{j}^{y}-J \sigma_{j}^{x} \sigma_{j+1}^{y}-J \sigma_{j-1}^{y} \sigma_{j}^{x} .
$$

Its squared norm is computed by using the $T=\infty$ limit of Kubo product, Eq. (6). The result is

$$
\left(f_{1}, f_{1}\right)=4 J^{2} \text {, }
$$

which already includes the average over the random fields. After a somewhat lengthy but straightforward calculation, we exactly determined the vectors $f_{2}, f_{3}, \ldots, f_{6}$. The results are rather involved and shall not be reported here.

The first recurrent is readily determined,

$$
\Delta_{1}=\frac{\left(f_{1}, f_{1}\right)}{\left(f_{0}, f_{0}\right)}=4 J^{2} .
$$

Notice that it does not depend on the external magnetic fields $B_{A}$ or $B_{B}$. The next recurrent is

$$
\Delta_{2}=5 J^{2}+2\left[p B_{A}^{2}+(1-p) B_{B}^{2}\right]-2\left[p B_{A}+(1-p) B_{B}\right]^{2},
$$

which now depends on both $B_{A}$ and $B_{B}$. By following this procedure, we also calculated $\Delta_{3}, \ldots, \Delta_{6}$. The results, however, are again too lengthy to be reproduced here. Rather, we present the results for the recurrents in Fig. 1. They are shown for $n \leqslant 6$ and different concentrations $p$ of $B_{A}=0$, and are valid in the high-temperature limit $T=\infty$. We notice that at $p=0.0(B=1.5 \mathrm{~J}$ along the whole chain) or $p=1.0$ (no external field) the system has the same recurrents. It means that in the case of either a uniform field or in the absence of a field, the dynamics will be the same, since the recurrents determine uniquely the time-dependent correlations. The cases $p=0.2$ and 0.8 also yield the same recurrents, and so do all the complementary concentrations $p$ and $1-p$. Such feature will be explained in the next section. 


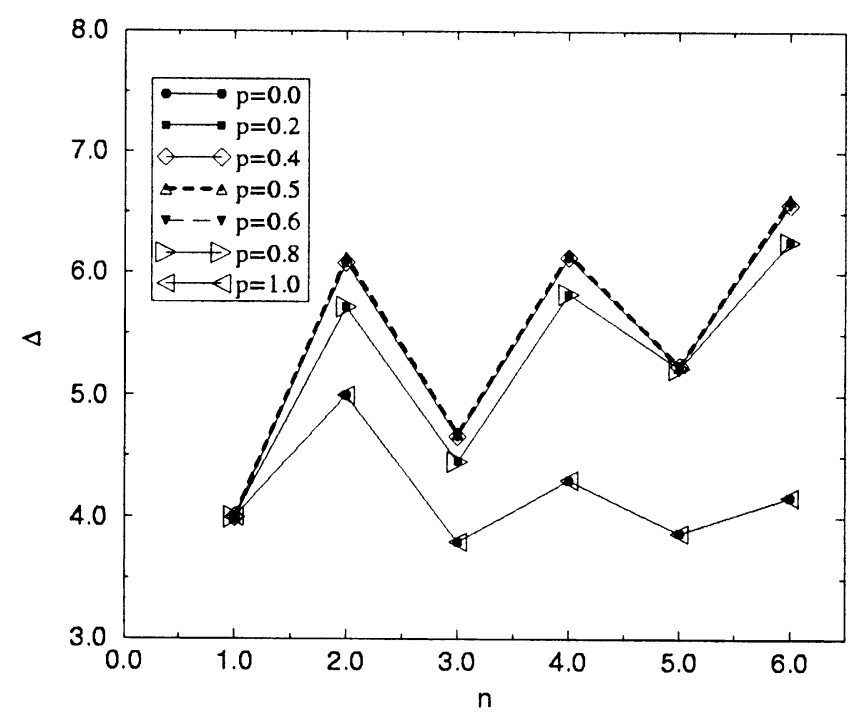

Fig. 1. Recurrants, in the infinite-temperature limit, for several values of $p$. In the figure, $B_{A}=0, B_{B}=1.5 J$, and $J=1$. The lines are just guides to the eye.

In order to determine the time-dependent autocorrelation function at $T=\infty$, defined by Eq. (12), one must have the moments $\mu_{2 k}$ or, equivalently, the recurrents $\Delta_{n}$. Usually, when just a few of the recurrents are available, one can only find the short-time expansion of $C(t)$. In order to extend the region of validity to longer times, we propose an ansatz for $\Delta_{n}, n>6$. Such approach relies strongly on the behavior of the known recurrents. For the isotropic $X Y$ chain in the absence of an applied field, it can be shown from the exact result of Ref. [10] that the recurrents tend to a finite value $\Delta_{\infty}=4 J^{2}$ as $n \rightarrow \infty$. In the present case, the results of Fig. 1 suggest that the recurrents also approach a finite value as $n$ gets larger, even in the disordered cases $0<p<1$ (recall that $p=0$ and 1 are disorderless cases). We shall assume that both the odd- and even-ordered recurrents approach a common terminal value $\Delta_{\infty}$ by following independent power-laws, just like in the case of the isotropic $X Y$ chain.

Based on the above considerations, we can construct an approximant to the higherorder recurrents in the infinite-temperature limit: (i) first, we estimate the value of $\Delta_{\infty}$ by plotting $\Delta_{n}$ versus $1 / n$ and assuming that $\Delta_{\infty}$ is given by its extrapolated value at the origin of the horizontal axis (as $n \rightarrow \infty$ ); (ii) next, we use the following ansatz for the high-ordered recurrents,

$$
\begin{aligned}
& \Delta_{n}=\frac{A}{n^{\eta}}+\Delta_{\infty}, \quad n=1,3,5, \ldots, \\
& A=\left(\Delta_{3}-\Delta_{\infty}\right) 3^{\eta}, \\
& \eta=-\ln \left(\frac{\Delta_{\infty}-\Delta_{5}}{\Delta_{\infty}-\Delta_{3}}\right) / \ln \left(\frac{5}{3}\right)
\end{aligned}
$$


Table 1

Parameters of the ansatz for the recurrents in the infinite-temperature limit, Eqs. (23)-(28). We used $B_{A}=0$, $B_{B}=1.5 J$, and $J=1$

\begin{tabular}{llllll}
\hline$p$ & $\Delta_{\infty}$ & $A$ & $\eta$ & $B$ & $\xi$ \\
\hline 0.0 and 1.0 & 4.15 & -0.58 & 0.45 & -0.58 & 0.45 \\
0.2 and 0.8 & 6.05 & -0.49 & 0.56 & -6.53 & 1.28 \\
0.6 and 0.4 & 6.34 & -0.33 & 0.28 & -4.16 & 0.83 \\
0.5 & 6.37 & -0.28 & 0.17 & -3.99 & 0.78 \\
\hline
\end{tabular}

and

$$
\begin{aligned}
& \Delta_{n}=\frac{B}{n^{\xi}}+\Delta_{\infty}, \quad n=2,4,6, \ldots, \\
& B=\left(\Delta_{4}-\Delta_{\infty}\right) 4^{\xi}, \\
& \xi=-\ln \left(\frac{\Delta_{\infty}-\Delta_{4}}{\Delta_{\infty}-\Delta_{6}}\right) / \ln \left(\frac{3}{2}\right) .
\end{aligned}
$$

The parameters $\Delta_{\infty}, A, \eta, B$, and $\xi$, which appear in the ansatz above, are listed in Table 1 for several values of $p$, for $B_{A}=0, B_{B}=1.5 J, J=1$, in the infinite-temperature limit.

It is worthwhile to compare the outcomes of our extrapolation scheme above with a known exact result, so that one can attest the reliability of the method. Consider the $X Y$ chain with isotropic interactions $J_{i}=J$, in the absence of external fields, in the infinite-temperature limit, that is, the system for which Niemeijer's solution applies. The recurrents $\Delta_{n}$, derived from that solution, $J_{0}(2 J t)^{2}$, oscillate about a terminal value $\Delta_{\infty}=4 J^{2}$ as $n$ grows, with decreasing amplitude. Both the odd and even recurrents ultimately reach $\Delta_{\infty}$ (at $\left.n=\infty\right)$ by following independent power laws. We need at least the first 60 exact recurrents to reconstruct $J_{0}(2 J t)^{2}$ in the time region $0 \leqslant t \leqslant 10$ (in units of $J^{-1}$ ), such that no visible difference can be seen in the scale of the figures used in this work. Extensions for larger $t$ can be obtained by using more recurrents. Now, the present approach yields $\Delta_{\infty}=4.15 J^{2}$ (see Table 1), which is marginally higher than the exact value. Thus, the recurrents from the ansatz tend to the terminal value with a slower power-laws of $n$, as compared to the exact recurrents. The implications of these on the time-dependent correlation function are shown in Fig. 2 which shows the results from our ansatz for the $X Y$ case in comparison to the exact solution. One can clearly see that, despite the fact that our approximation is based on six exact recurrents only, we obtain good quantitative results for times up to $t=4.5$. The behavior of the approximation for $t \geqslant 4.5$ has the same general behavior as the exact one, except that $C(t)$ is slightly shifted upward, causing it never to vanish at the zeros of $J_{0}(2 J t)$, as the exact result does. In addition, the approximate $C(t)$ seems to tend to a finite, albeit small, value as $t \rightarrow \infty$, as opposed to exact result, which vanishes at that limit. Hence, based on the above considerations, we are led to expect that our results for the cases with disorder should behave as nearly exactly at short times, and be a little overestimated at longer times. Finally, since the results for $p=0$ and 1 coincide with 


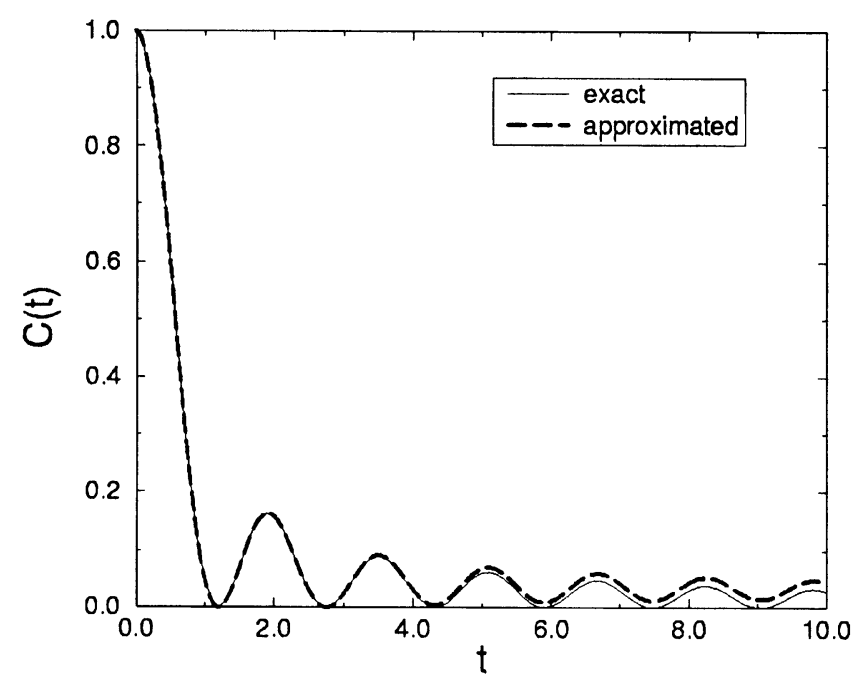

Fig. 2. Comparison between exact and approximate autocorrelation function for the pure $X Y$ model with or without a uniform field (i.e., $p=0$ or $p=1$ ) at $T=\infty$. Both curves were drawn by considering the first 60 recurrents only.

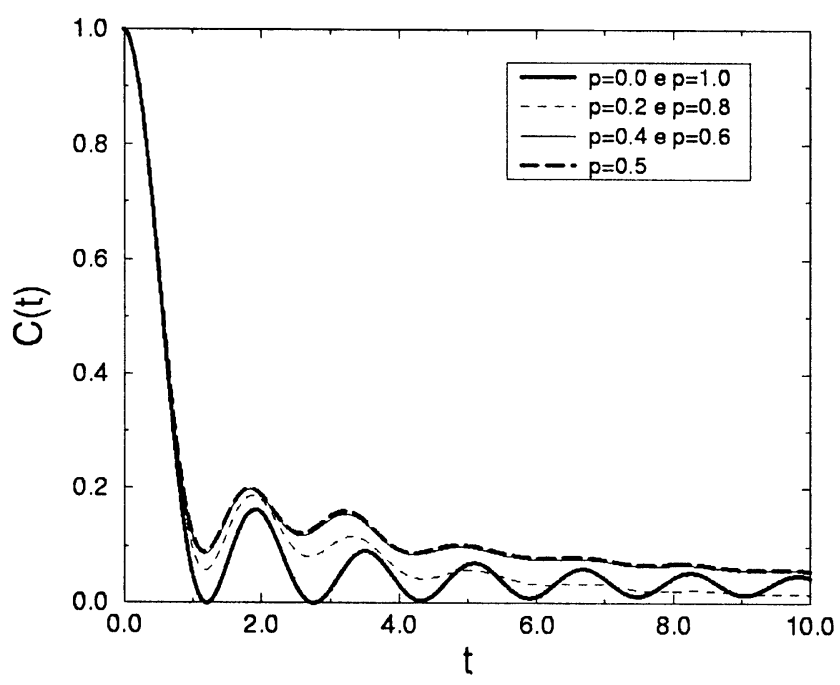

Fig. 3. Time-dependent autocorrelation function of the $X Y$ chain in the infinite-temperature limit for several values of $p$, where we use $B_{A}=0, B_{B}=1.5 J(J=1)$.

each other, as shown in Fig. 2, we conclude that the presence or not of a uniform field does not alter the autocorrelation function.

Figs. 3 and 4 show the time-dependent autocorrelation function and the corresponding spectral densities, respectively, for several distribution of fields. Disorder lifts $C(t)$ 


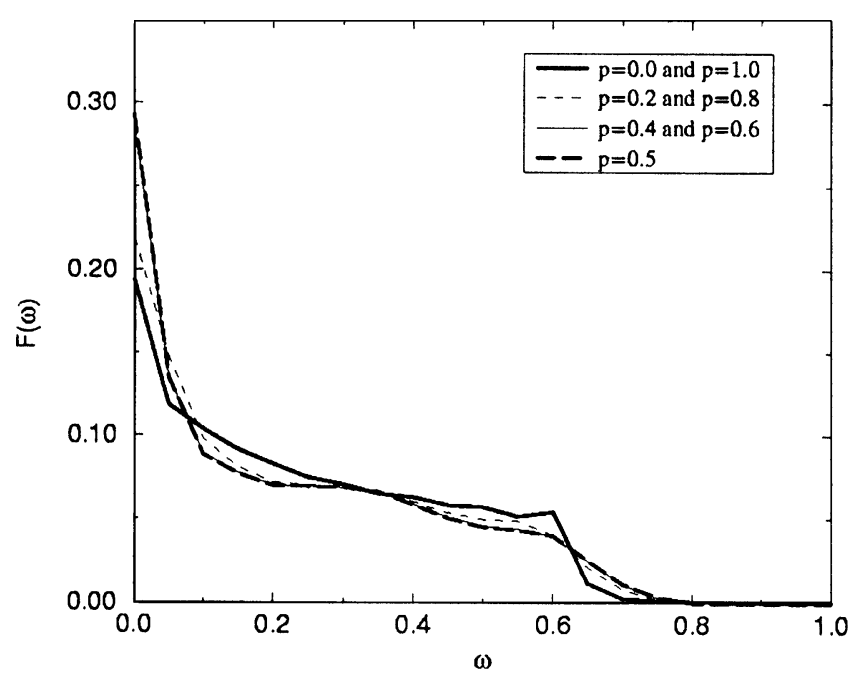

Fig. 4. Spectral density function of the $X Y$ chain in the limit of high temperature, $T=\infty$, for several values of $p$. Here, $B_{A}=0, B_{B}=1.5 \mathrm{~J}$, and $J=1$.

appreciably, thereby producing slower decay at large $t$. Such effect is most pronounced at maximum disorder, $p=0.5$. The disorder-induced raise of $C(t)$ is already noticeable at short times, where the curves are essentially exact. The system presents an interesting behavior when a random external field is applied: it is susceptible to the percentage of disorder, but not to the intensity of the field. For example, the cases $p=0.2$ and 0.8 have the same behavior, although the percentage of sites under the effect of external field is different. Similar results are obtained for other values of $B_{A}$ and $B_{B}$. The explanation is given in next section, since it also applies to the case of a ladder.

\section{Dynamics of the $X Y$-Ising ladder in a random field}

We now consider the dynamics of the two-leg spin ladder on a random field in the high-temperature limit, $T=\infty$. We set $J_{x}=J_{y}=J$ and $J_{z}=0$ in Hamiltonian (2). The external fields act on sites of both legs of the ladder. We consider two independent random field variables, $B_{i, 1}$ and $B_{i, 2}$, distributed according to

$$
\begin{aligned}
& \rho\left(\left\{B_{i, 1}\right\}\right)=\prod_{i}^{N}\left[q \delta\left(B_{i, 1}-B_{A}\right)+(1-q) \delta\left(B_{i, 1}-B_{B}\right)\right], \\
& \rho\left(\left\{B_{i, 2}\right\}\right)=\prod_{i}^{N}\left[r \delta\left(B_{i, 2}-B_{C}\right)+(1-r) \delta\left(B_{i, 2}-B_{D}\right)\right],
\end{aligned}
$$

where $0 \leqslant q, r \leqslant 1$. The quantities $B_{A}$ and $B_{B}$ are random fields on chain 1 , while $B_{C}$ and $B_{D}$ are the random fields on chain 2, respectively. We use the same method 


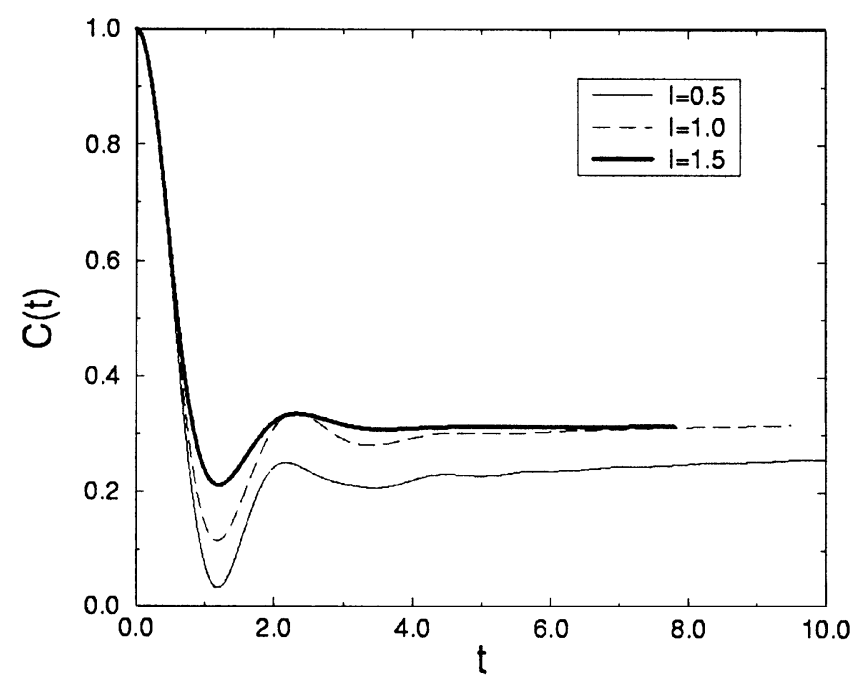

Fig. 5. Autocorrelation function of the $X Y$ model on a ladder without external field $\left(B_{A}=B_{B}=B_{C}=B_{D}=0\right)$ and isotropic exchange interactions, $J=1$, for a few values of the Ising interchain coupling $I$ (in units of $J$ ), in the infinite-temperature limit.

as in Section 3 to investigate the longitudinal dynamic spin autocorrelation functions on the ladder, $C(t)=\left\langle\sigma_{j}^{z}(0) \sigma_{j}^{z}(t)\right\rangle$. We analytically determine the first four recurrents only, since the higher connectivity of the ladder makes the calculations much longer than those of the chain. We then use a similar procedure as in the previous section to extrapolate to longer times $t$. Next, we devise an ansatz similar to Eqs. (23)-(28), to estimate the next 56 recurrents. We do not present the details here since the ansatz can be easily reproduced by following the steps outlined in the last section. The only difference is that now we have only four exact recurrents, thus making the ensuing extrapolation less reliable. Nevertheless, as we shall see, the method works remarkably well and we obtain sensible results for the autocorrelation functions.

We now outline the results for the time-dependent autocorrelation functions in the $T=\infty$ limit, shown in Figs. 5 and 6 . The case without external field is depicted in Fig. 5. It shows the effects of the competition between the $X Y$ coupling along the legs and the Ising interchain coupling on the time-dependent longitudinal correlation function. The $X Y$ coupling favors a $\left[J_{0}(2 J t)\right]^{2}$-type of behavior, Niemeijer's solution for the chain, while the Ising coupling drives the correlation function towards unity, which is the case for Ising interactions only. For very low $I$, the dynamics is mainly of the $X Y$-chain type, since the two legs are almost disconnected. As $I$ increases, the oscillations pertaining to the $X Y$ behavior are still present, but the Ising interaction lifts the curves $C(t)$ above the time axis. In the limit $I \gg J$, the correlation function will approach unity for all $t$, since the $z$-component of spin is then essentially a constant of motion. Hence, for a given value of $I$ in the long-time limit $C(t)$ will approach a constant value between 0 and 1 , corresponding to the extreme cases of couplings $I=0$ 


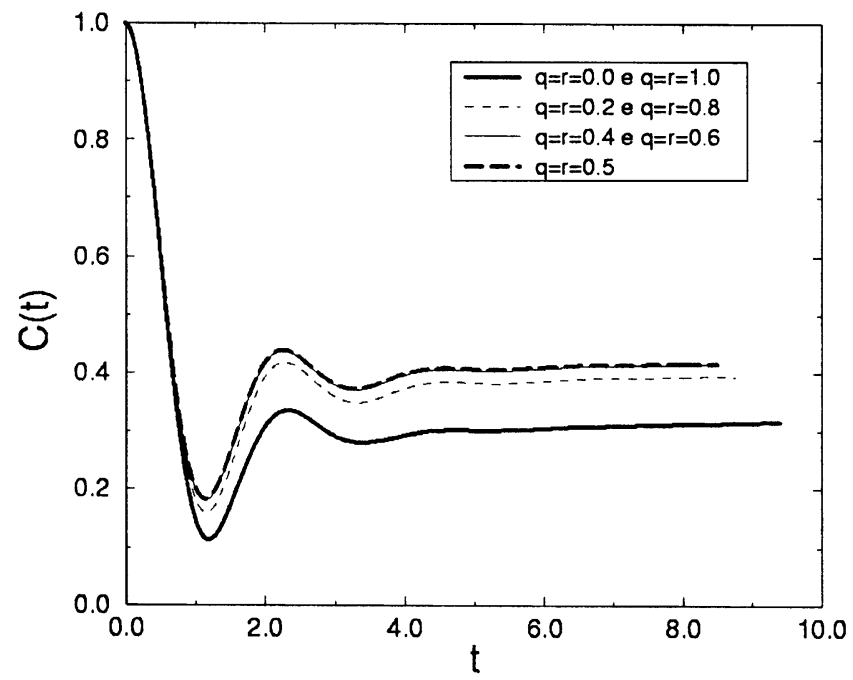

Fig. 6. Autocorrelation function for the $X Y$ model on a ladder with disordered fields $\left(B_{A}=B_{C}=0\right.$ and $\left.B_{B}=B_{D}=1.5 J\right)$ for $J_{i, 1}=J_{i, 2}=J=I=1$, at $T=\infty$.

and $\infty$. One should bear in mind that the long-time behavior shown in Fig. 5 is very likely to be a bit overestimated due to same reasons described in the last section. For example, the steady rise in $C(t)$ at large $t$ is surely an artifact of the ansatz used. Still our conclusions above should hold true, since the raises of $C(t)$ due to uncertainties in the ansatz, are much smaller than the effects of the interchain coupling, especially from moderate $I \sim J$ to higher couplings $I>J$.

Fig. 6 shows $C(t)$, in the infinite-temperature limit, for different concentrations of equal fields on each chain (as well as equal concentrations on each chain). The couplings $J=I$, while the fields $B_{i}$ are disordered and obey bimodal distributions, with probabilities $q$ and $r$ for zero field, otherwise $B_{i}=1.5 \mathrm{~J}$. We can see that, as in the case of the single chain model, the presence or not of an external field produces the same dynamic behavior for the disorderless cases $(p=0$ and 1). The same holds true for the symmetric pairs of distributions $(q, 1-q)$ and $(r, 1-r)$. Such result is due to the up-down symmetry of the model Hamiltonian along the $z$-direction. This symmetry is best seen with the canonical transformation of $\sigma_{i}^{x} \rightarrow \sigma_{i}^{y}, \sigma_{i}^{y} \rightarrow \sigma_{i}^{x}, \sigma_{i}^{z} \rightarrow-\sigma_{i}^{z}$, which changes the sign of the interaction energy with the longitudinal field and, of course, leaves the energy spectrum unchanged. Hence, a field applied to either the positive or negative direction of the $z$-axis will yield the same results for both the static and dynamic quantities. Suppose we exchange the probabilities $q$ and $1-q$, as well as $r$ and $1-r$ from the distributions of fields. This is equivalent to the exchange of the end values of the fields in the bimodal distributions. The form of the new Hamiltonian will be the same as that of the canonically transformed Hamiltonian, apart from a shift in the overall energy scale, which does not affect the dynamics. Therefore, the dynamic correlations of the cases $(q, 1-q)$ and $(r, 1-r)$ should be identical, as our results 
show in Fig. 6. These considerations are quite general and apply to any space dimensions. The Ising coupling $I=J$ already leads to non-zero long-time behavior for $C(t)$, however disorder also lifts $C(t)$, with highest values at maximum disorder, $q=r=0.5$. Similar results are obtained for different values of the Ising interchain coupling $I$.

\section{Conclusions}

We investigated the time evolution of the spin- $\frac{1}{2} X Y$ isotropic model defined on a single chain and on a two-leg ladder (with Ising-type interchain couplings), at infinite temperature, by using the method of recurrence relations. We exactly determined the first six moments (for the model on a chain) and the first four moments (for the model on a ladder) of the time-dependent longitudinal correlations in the high-temperature limit. We extended the exact results, which are valid for short times only, by using an approximate method in which the recurrents are assumed to oscillate towards a terminal value $\Delta_{\infty}$. Within this scheme, we were able to reproduce fairly well-known exact results, such as the $X Y$ chain without applied field. The most interesting aspect of the present results is the dependence of the chain and the ladder under a disordered field. Both are sensible to the percentage of disorder, but not to the intensity of the fields. Despite its simplicity in using just a few exact recurrents, the present approach is able to give a good picture of the dynamics of the $X Y$ chain and ladder in the presence of random fields.

\section{Acknowledgements}

This work was partially supported by CNPq, FAPEMIG, and PRONEX (Brazilian agencies).

\section{References}

[1] E. Lieb, T. Schultz, D.C. Mattis, Ann. Phys. (N.Y.) 16 (1961) 407.

[2] S. Katsura, Phys. Rev. 127 (1962) 1508.

[3] S. Katsura, T. Horiguchi, M. Suzuki, Physica 46 (1970) 67.

[4] J.H.H. Perk, H.W. Capel, Physica 89 A (1977) 265.

[5] E. Goovaerts, H. De Raedt, D. Schoemaker, Phys. Rev. Lett. 52 (1984) 1649.

[6] M. Mohan, G. Müller, Phys. Rev. B 27 (1983) 1776.

[7] P. Jordan, E. Wigner, Z. Phys. 47 (1928) 631.

[8] M. Suzuki, J. Phys. Soc. Jpn. 21 (1966) 2140.

[9] O. Derzhko, J. Richter, Phys. Rev. B 55 (1977) 14298.

[10] Th. Niemeijer, Physica 36 (1967) 377;

Th. Niemeijer, Physica 39 (1968) 313.

[11] B.M. McCoy, Phys. Rev. 173 (1968) 531.

[12] E. Barouch, B.M. McCoy, M. Dresden, Phys. Rev. A 2 (1970) 1075.

[13] E. Barouch, B.M. McCoy, Phys. Rev. A 3 (1971) 786;

E. Barouch, B.M. McCoy, Phys. Rev. A 3 (1971) 2137.

[14] E. Barouch, B.M. McCoy, D.B. Abraham, Phys. Rev. A 4 (1971) 2331. 
[15] A. Sur, D. Jasnow, I.L. Lowe, Phys. Rev. B 12 (1975) 3845.

[16] U. Brandt, K. Jacoby, Z. Phys. B 25 (1976) 181.

[17] H.W. Capel, J.H.H. Perk, Physica 87 A (1977) 211.

[18] J. Florencio, M.H. Lee, Phys. Rev. B 35 (1987) 1835.

[19] D.C. Johnston, J.W. Johnston, D.P. Goshorn, A.J. Jacobson, Phys. Rev. B 35 (1987) 219.

[20] M. Hagiwara, H.A. Katori, U. Schollwöck, H.J. Mikeska, Phys. Rev. B 62 (2000) 1051.

[21] L. Hubert, A. Caillé, Phys. Rev. B 43 (1991) 13,187.

[22] T. Hikihara, A. Furusaki, Phys. Rev. B 63 (2001) 1,34,438.

[23] E. Orignac, T. Giamarchi, Phys. Rev. B 57 (1998) 5812.

[24] R. Mélin, Y.C. Lin, P. Lajkó, H. Rieger, F. Iglói, Phys. Rev. B 65 (2002) 1,04,415.

[25] M.H. Lee, Phys. Rev. Lett. 49 (1982) 1072;

M.H. Lee, Phys. Rev. B 26 (1982) 2547;

M.H. Lee, J. Math. Phys. 24 (1983) 2512.

[26] M.H. Lee, J. Hong, J. Florencio, Physica Scripta T19 (1987) 498.

[27] M.H. Lee, Phys. Rev. E 61 (2000) 3571.

[28] M.H. Lee, Phys. Rev. E 62 (2000) 1769.

[29] J. Florencio, S. Sen, M.H. Lee, Braz. J. Phys. 30 (2000) 725.

[30] M.H. Lee, Phys. Rev. Lett. 51 (1983) 1227.

[31] J. Hong, M.H. Lee, Phys. Rev. Lett. 55 (1985) 2375.

[32] J. Hong, M.H. Lee, Phys. Rev. Lett. 70 (1993) 1972.

[33] J. Florencio, O.F. de Alcantara Bonfim, Phys. Rev. Lett. 72 (1994) 3286

[34] J. Florencio, S. Sen, Z.X. Cai, J. Phys.: Condens. Matter 7 (1995) 1363.

[35] J. Florencio, F.C. Sá Barreto, Phys. Rev. B 60 (1999) 9555.

[36] J. Florencio, M.H. Lee, Phys. Rev. A 31 (1985) 3231.

[37] I. Sawada, Phys. Rev. Lett. 83 (1999) 1668.

[38] J. Kim, I. Sawada, Phys. Rev. E 61 (2000) R2172.

[39] U. Balucani, M.H. Lee, V. Tognetti, Phys. Rep. 373 (2003) 409.

[40] M.H. Lee, Phys. Rev. Lett. 87 (2001) 25061

[41] R. Kubo, J. Phys. Soc. (Jpn) 12 (1957) 570;

R. Kubo, Rep. Prog. Phys. 29 (1966) 225. 